PROCEEDINGS OF THE

AMERICAN MATHEMATICAL SOCIETY

Volume 129, Number 11, Pages 3339-3344

S 0002-9939(01)06130-5

Article electronically published on April 9, 2001

\title{
AN OPERATOR INEQUALITY RELATED TO JENSEN'S INEQUALITY
}

\author{
MITSURU UCHIYAMA
}

(Communicated by Joseph A. Ball)

Abstract. For bounded non-negative operators $A$ and $B$, Furuta showed

$$
0 \leq A \leq B \text { implies } A^{\frac{r}{2}} B^{s} A^{\frac{r}{2}} \leq\left(A^{\frac{r}{2}} B^{t} A^{\frac{r}{2}}\right)^{\frac{s+r}{t+r}} \quad(0 \leq r, 0 \leq s \leq t) .
$$

We will extend this as follows: $0 \leq A \leq B !{ }_{\lambda} C(0<\lambda<1)$ implies

$$
A^{\frac{r}{2}}\left(\lambda B^{s}+(1-\lambda) C^{s}\right) A^{\frac{r}{2}} \leq\left\{A^{\frac{r}{2}}\left(\lambda B^{t}+(1-\lambda) C^{t}\right) A^{\frac{r}{2}}\right\}^{\frac{s+r}{t+r}},
$$

where $B ! C$ is a harmonic mean of $B$ and $C$. The idea of the proof comes from

Jensen's inequality for an operator convex function by Hansen-Pedersen.

\section{INTRODUCTION}

Throughout this article, an operator means a bounded linear operator on a Hilbert space. For selfadoint operators $A, B$ we write $A \leq B$ as usual if $B-A$ is positive semidefinite. A real continuous function $f$ defined on an interval $I$ is said to be operator monotone if $f$ preserves this order, that is, for bounded selfadjoint operators $A, B$ with spectra in $I$,

$$
A \leq B \quad \Rightarrow \quad f(A) \leq f(B)
$$

and it is said to be operator convex if for all selfadjoint operators $A, B$ with spectra in $I$ and for all $\lambda$ in $[0,1]$

$$
f(\lambda A+(1-\lambda) B) \leq \lambda f(A)+(1-\lambda) f(B) .
$$

An operator concave function is similarly defined. In [6] Hansen and Pedersen showed that for a non-negative continuous function $f$ on $[0, \infty)$ the following conditions are equivalent:

(i) $f$ is operator monotone,

(ii) $f$ is operator concave,

(iii) $T^{*} f(A) T \leq f\left(T^{*} A T\right)$ for every contraction $T$ (i.e., $\|T\| \leq 1$ ) and for every non-negative operator $A$,

(iv) $S^{*} f(A) S+T^{*} f(B) T \leq f\left(S^{*} A S+T^{*} B T\right)$ for every pair of $S, T$ with $S^{*} S+$ $T^{*} T \leq 1$ and for all non-negative operators $A, B$.

Received by the editors March 21, 2000.

2000 Mathematics Subject Classification. Primary 47A63, 15A48.

Key words and phrases. Order of selfadjoint operators, Jensen inequality, Furuta inequality. 
It is well-known that $f(x)=x^{a} \quad(0<a \leq 1)$ is operator monotone on $[0, \infty)$, that is,

$$
0 \leq A \leq B \Rightarrow A^{a} \leq B^{a}
$$

which is called the Löwner-Heinz inequality. Therefore, (iv) yields

$$
S^{*} A^{a} S+T^{*} B^{a} T \leq\left(S^{*} A S+T^{*} B T\right)^{a} \quad(0<a \leq 1) .
$$

Related to the Löwner-Heinz inequality, Furuta showed (cf. [5]): for non-negative real numbers $r, s$ and $t$ such that $t \geq s$ and $(r, t) \neq(0,0)$,

$$
0 \leq A \leq B \Rightarrow A^{\frac{r}{2}} B^{s} A^{\frac{r}{2}} \leq\left(A^{\frac{r}{2}} B^{t} A^{\frac{r}{2}}\right)^{\frac{s+r}{t+r}}, \quad\left(B^{\frac{r}{2}} A^{t} B^{\frac{r}{2}}\right)^{\frac{s+r}{t+r}} \leq B^{\frac{r}{2}} A^{s} B^{\frac{r}{2}} .
$$

For non-negative operators $A$ and $B$ and for a real number $\lambda$ with $0<\lambda<1$, the harmonic mean is defined by

$$
A ! B:=\left(\lambda A^{-1}+(1-\lambda) B^{-1}\right)^{-1}
$$

if $A$ and $B$ are invertible, and defined by the weak limit of $(A+\epsilon) \underset{\lambda}{!}(B+\epsilon)$ as $\epsilon \rightarrow+0$ if not. If non-negative operators $A, B$ are invertible, then we have

$\lambda A^{-1}+\mu B^{-1}-(\lambda A+\mu B)^{-1}=\left(A^{-1}-B^{-1}\right)\left((\lambda A)^{-1}+(\mu B)^{-1}\right)^{-1}\left(A^{-1}-B^{-1}\right)$, where $0 \leq \lambda, \mu \leq 1, \lambda+\mu=1$ (see [10, p. 117 of [2]). This shows that a function $f(x)=1 / x$ is operator convex on $(0, \infty)$ and that

$$
A ! B \leq \lambda A+(1-\lambda) B .
$$

We need the following properties of the harmonic mean (cf. [1], [7]):

$$
\begin{aligned}
& (\alpha A) !(\alpha B)=\alpha(A ! B), \\
& A ! B \stackrel{\lambda}{\leq} \leq{ }_{\lambda} ! B \text { if } A \stackrel{\lambda}{\leq} C \text {, and }
\end{aligned}
$$

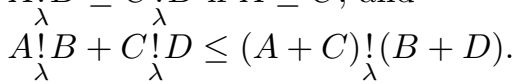

\section{MAIN TheOREM}

From now on, $\lambda$ and $\mu$ represent real numbers such that

$$
0 \leq \lambda, \mu \leq 1 \text { and } \lambda+\mu=1
$$

We start this section with a simple inequality.

Lemma 2.1. Let $S, T$ be contractions such that $S^{*} S+T^{*} T \leq 1$, and let $A, B$ be non-negative operators. Then for $0 \leq r \leq s \leq t$ and $s \neq 0$,

$$
\begin{aligned}
\left(S^{*} A^{s} S+T^{*} B^{s} T\right) & \leq\left(S^{*} A^{t} S+T^{*} B^{t} T\right)^{\frac{s}{t}}, \\
\left(S^{*} A^{s} S+T^{*} B^{s} T\right)^{\frac{r}{s}} & \leq\left(S^{*} A^{t} S+T^{*} B^{t} T\right)^{\frac{r}{t}} .
\end{aligned}
$$

Proof. The first inequality follows from (1). By using the Löwner-Heinz inequality we get the second inequality.

We remark that the above implies that for fixed $r>0$ the operator valued function $\left(S^{*} A^{t} S+T^{*} B^{t} T\right)^{\frac{r}{t}}$ is increasing on $r \leq t<\infty$.

Lemma 2.2. Let $H$ and $K$ be bounded selfadjoint operators such that $0 \leq H \leq K$. Then for real numbers $p, q$ such that $0 \leq p \leq q$

$$
H^{\frac{1}{2}} K^{p} H^{\frac{1}{2}} \leq\left(H^{\frac{1}{2}} K^{q} H^{\frac{1}{2}}\right)^{\frac{p+1}{q+1}}, \quad K^{\frac{1}{2}} H^{p} K^{\frac{1}{2}} \geq\left(K^{\frac{1}{2}} H^{q} K^{\frac{1}{2}}\right)^{\frac{p+1}{q+1}} .
$$


Proof. To show the first inequality we may assume that $K$ is invertible. Since $\left\|K^{-\frac{1}{2}} H^{\frac{1}{2}}\right\| \leq 1$, by Lemma 2.1 , we get

$$
\left(H^{\frac{1}{2}} K^{-\frac{1}{2}} K^{p+1} K^{-\frac{1}{2}} H^{\frac{1}{2}}\right) \leq\left(H^{\frac{1}{2}} K^{-\frac{1}{2}} K^{q+1} K^{-\frac{1}{2}} H^{\frac{1}{2}}\right)^{\frac{p+1}{q+1}} .
$$

This gives the first inequality. By considering the inverse of $K$ and $H$, the second inequality follows from the first one.

Now we give the main theorem that is an extension of (2).

Theorem 2.3. Let $A, B$ and $C$ be non-negative operators. Then for non-negative real numbers $r, s, t$ such that $s \leq t$ and $(r, t) \neq(0,0)$,

$$
A \leq B !{ }_{\lambda} C \Longrightarrow A^{\frac{r}{2}}\left(\lambda B^{s}+\mu C^{s}\right) A^{\frac{r}{2}} \leq\left\{A^{\frac{r}{2}}\left(\lambda B^{t}+\mu C^{t}\right) A^{\frac{r}{2}}\right\}^{\frac{s+r}{t+r}} .
$$

Proof. Suppose $r=0$. Then, since $f(x)=x^{s / t}$ is operator concave, we obtain (3). Thus, we need to show (3) in the case of $r>0$. Since $A \leq(B+\epsilon) !(C+\epsilon)$, we may assume that $B$ and $C$ are invertible. We first suppose $0<r \leq 1$. Since $f(x)=x^{r}$ is operator monotone and operator concave,

$$
A \leq\left(\lambda B^{-1}+\mu C^{-1}\right)^{-1}
$$

yields

$$
A^{r} \leq\left(\lambda B^{-1}+\mu C^{-1}\right)^{-r} \leq\left(\lambda B^{-r}+\mu C^{-r}\right)^{-1} .
$$

This implies

$$
A^{\frac{r}{2}}\left(\lambda B^{-r}+\mu C^{-r}\right) A^{\frac{r}{2}} \leq 1
$$

By Lemma 2.1, for $0 \leq s \leq t$ we get

$$
\begin{aligned}
A^{\frac{r}{2}}\left(\lambda B^{s}+\mu C^{s}\right) A^{\frac{r}{2}} & =\lambda A^{\frac{r}{2}} B^{-\frac{r}{2}} B^{s+r} B^{-\frac{r}{2}} A^{\frac{r}{2}}+\mu A^{\frac{r}{2}} C^{-\frac{r}{2}} C^{s+r} C^{-\frac{r}{2}} A^{\frac{r}{2}} \\
& \leq\left(\lambda A^{\frac{r}{2}} B^{-\frac{r}{2}} B^{t+r} B^{-\frac{r}{2}} A^{\frac{r}{2}}+\mu A^{\frac{r}{2}} C^{-\frac{r}{2}} C^{t+r} C^{-\frac{r}{2}} A^{\frac{r}{2}}\right)^{\frac{s+r}{t+r}} \\
& =\left(\lambda A^{\frac{r}{2}} B^{t} A^{\frac{r}{2}}+\mu A^{\frac{r}{2}} C^{t} A^{\frac{r}{2}}\right)^{\frac{s+r}{t+r}} .
\end{aligned}
$$

This means (3) holds for $0<r \leq 1$ and for $0 \leq s \leq t$.

Assume (3) holds for $0<r \leq 2^{n}$ and for $0 \leq s \leq t$. Take an arbitrary $r$ in $\left(2^{n}, 2^{n+1}\right]$. Since $r / 2 \leq 2^{n}$, the assumption says

$$
A^{\frac{r}{4}}\left(\lambda B^{s}+\mu C^{s}\right) A^{\frac{r}{4}} \leq\left\{A^{\frac{r}{4}}\left(\lambda B^{t}+\mu C^{t}\right) A^{\frac{r}{4}}\right\}^{\frac{s+r / 2}{t+r / 2}} \quad(0 \leq s \leq t) ;
$$

in particular,

$$
A^{\frac{r}{2}} \leq\left\{A^{\frac{r}{4}}\left(\lambda B^{t}+(1-\lambda) C^{t}\right) A^{\frac{r}{4}}\right\}^{\frac{r}{2 t+r}} .
$$

Let us apply this to the first inequality of Lemma 2.2 with $p=(2 s+r) / r, q=$ $(2 t+r) / r$. Then we get

$$
A^{\frac{r}{4}}\left\{A^{\frac{r}{4}}\left(\lambda B^{t}+(1-\lambda) C^{t}\right) A^{\frac{r}{4}}\right\}^{\frac{2 s+r}{2 t+r}} A^{\frac{r}{4}} \leq\left[A^{\frac{r}{4}}\left\{A^{\frac{r}{4}}\left(\lambda B^{t}+(1-\lambda) C^{t}\right) A^{\frac{r}{4}}\right\} A^{\frac{r}{4}}\right]^{\frac{s+r}{t+r}} .
$$

This in conjunction with (4) gives

$$
A^{\frac{r}{2}}\left(\lambda B^{s}+(1-\lambda) C^{s}\right) A^{\frac{r}{2}} \leq\left\{A^{\frac{r}{2}}\left(\lambda B^{t}+(1-\lambda) C^{t}\right) A^{\frac{r}{2}}\right\}^{\frac{s+r}{t+r}} .
$$

Corollary 2.4. Let $A, B$ and $C$ be non-negative operators. Then

$$
A \leq B ! \dot{\lambda}^{\prime} C A^{1+r} \leq\left\{A^{\frac{r}{2}}\left(\lambda B^{t}+(1-\lambda) C^{t}\right) A^{\frac{r}{2}}\right\}^{\frac{1+r}{t+r}} \quad(1 \leq t) .
$$


Proof. Putting $s=1$ in (3), in virtue of $A \leq B !{ }_{\lambda} C \leq \lambda B+\mu C$, we get the above.

Considering the previous inequality with $B=C$, we get

$$
0 \leq A \leq B \Longrightarrow A^{1+r} \leq\left(A^{\frac{r}{2}} B^{t} A^{\frac{r}{2}}\right)^{\frac{1+r}{t+r}}
$$

This has been found by Furuta [4] and called the Furuta inequality. Furuta showed (2) by using (5). Our proof seems to help us clear up the significance of the exponent $(s+r) /(t+r)$ in $(2)$ and hence the exponent $(1+r) /(t+r)$ in (5) (cf. [8]).

If $A \leq B$ and $A \leq C$, then $A \leq B ! C$ for every $\lambda$; therefore, the inequality in (3) holds. We remark that in this case we can show it by (2) and the concavity of $f(x)=x^{(s+r) /(t+r)}$.

We proved that Theorem 2.3 and Corollary 2.4 hold for just the harmonic mean of two operators $B$ and $C$; however, it is easy to see that they do even for the harmonic mean of a finite number of operators, too.

Proposition 2.5. Let $B, C$ and $D$ be non-negative operators. Then for non-negative real numbers $r, s, t$ such that $s \leq t$ and $(r, t) \neq(0,0)$,

$$
\lambda B+\mu C \leq D \Rightarrow\left\{D^{\frac{r}{2}}\left(B^{t} ! C^{t}\right) D^{\frac{r}{2}}\right\}^{\frac{s+r}{t+r}} \leq D^{\frac{r}{2}}\left(B^{s} ! C^{s}\right) D^{\frac{r}{2}} .
$$

Proof. By the continuity of harmonic mean in the norm sense, we may assume that $B, C$ and $D$ are all invertible. The condition $\lambda B+\mu C \leq D$ implies

$$
D^{-1} \leq B^{-1} ! C^{-1}
$$

By Theorem 2.3, this yields

$$
D^{-\frac{r}{2}}\left(\lambda B^{-s}+\mu C^{-s}\right) D^{-\frac{r}{2}} \leq\left\{D^{-\frac{r}{2}}\left(\lambda B^{-t}+\mu C^{-t}\right) D^{-\frac{r}{2}}\right\}^{\frac{s+r}{t+r}} .
$$

Take the inverse of both sides of this inequality to get the required inequality.

Put $s=1$ in Proposition 2.5. Since

$$
B \underset{\lambda}{!} C \leq \lambda B+(1-\lambda) C \leq D,
$$

we get

Corollary 2.6. Let $B, C$ and $D$ be non-negative operators. Then for $0 \leq r$ and $1 \leq t$,

$$
\lambda B+\mu C \leq D \quad \Rightarrow \quad\left\{D^{\frac{r}{2}}\left(B^{t} ! C^{t}\right) D^{\frac{r}{2}}\right\}^{\frac{1+r}{t+r}} \leq D^{1+r}
$$

By setting $B=C$ in the above, we get an alternate Furuta inequality:

$$
0 \leq C \leq D \Rightarrow\left(D^{\frac{r}{2}} C^{t} D^{\frac{r}{2}}\right)^{\frac{1+r}{t+r}} \leq D^{1+r} .
$$

By combining (5) and $(5)^{\prime}$, we get, for $0 \leq r, 1 \leq t$,

$$
0 \leq A \leq B \leq C \Rightarrow\left(B^{\frac{r}{2}} A^{t} B^{\frac{r}{2}}\right)^{\frac{1+r}{t+r}} \leq B^{1+r} \leq\left(B^{\frac{r}{2}} C^{t} B^{\frac{r}{2}}\right)^{\frac{1+r}{t+r}} .
$$

Suppose that $B$ and $C$ are non-negative operators. Replace $A$ with $B ! C$ in Theorem 2.3 and $D$ with $\lambda B+\mu C$ in Proposition 2.5. Then we get 
Corollary 2.7. Let $B, C$ be non-negative operators. Then for non-negative numbers $r, s, t$ such that $s \leq t$ and $(r, t) \neq(0,0)$,

$$
\begin{aligned}
& (B \underset{\lambda}{!} C)^{\frac{r}{2}}\left(\lambda B^{s}+\mu C^{s}\right)(B \underset{\lambda}{!} C)^{\frac{r}{2}} \leq\left\{(B ! C)^{\frac{r}{2}}\left(\lambda B^{t}+\mu C^{t}\right)\left(B{ }_{i} C\right)^{\frac{r}{2}}\right\}^{\frac{s+r}{t+r}} \\
& \left\{(\lambda B+\mu C)^{\frac{r}{2}}\left(B_{i}^{t} ! C^{t}\right)(\lambda B+\mu C)^{\frac{r}{2}}\right\}^{\frac{s+r}{t+r}} \leq(\lambda B+\mu C)^{\frac{r}{2}}\left(B_{i}^{s} C^{s}\right)(\lambda B+\mu C)^{\frac{r}{2}} .
\end{aligned}
$$

\section{EXPONENTIAL INEQUALITY}

Theorem 3.1. Let $A, B$ and $C$ be non-negative operators. Then for non-negative real numbers $r, s, t$ such that $s \leq t$ and $(r, t) \neq(0,0)$,

$$
A \leq B \underset{\lambda}{!} C \quad \Longrightarrow \quad e^{\frac{r}{2} A}\left(\lambda e^{s B}+\mu e^{s C}\right) e^{\frac{r}{2} A} \leq\left\{e^{\frac{r}{2} A}\left(\lambda e^{t B}+\mu e^{t C}\right) e^{\frac{r}{2} A}\right\}^{\frac{s+r}{t+r}} .
$$

Proof. For every natural number $n, 1+A / n \leq(1+B / n) !(1+C / n)$ because of the properties of harmonic mean given in Section 1.

Therefore, (3) gives

$$
\begin{aligned}
& (1+A / n)^{\frac{n r}{2}}\left\{\lambda(1+B / n)^{n s}+\mu(1+C / n)^{n s}\right\}(1+A / n)^{\frac{n r}{2}} \\
\leq & {\left[(1+A / n)^{\frac{n r}{2}}\left\{\lambda(1+B / n)^{n t}+\mu(1+C / n)^{n t}\right\}(1+A / n)^{\frac{n r}{2}}\right]^{\frac{n s+n r}{n t+n r}} . }
\end{aligned}
$$

Letting $n \rightarrow \infty$, we get the required inequality.

Remark. Harmonic mean is just defined for non-negative operators. However, it is clear that the above inequality holds for general selfadjoint operators $A, B, C$ if there is a real number $a$ so that $A+a, B+a, C+a \geq 0$ and $A+a \leq(B+a) !(C+a)$. In particular, putting $B=C$ we can obtain:

Let $A, B$ be (not necessarily non-negative) selfadjoint operators. Then

$$
A \leq B \Rightarrow e^{\frac{r}{2} A} e^{s B} e^{\frac{r}{2} A} \leq\left(e^{\frac{r}{2} A} e^{t B} e^{\frac{r}{2} A}\right)^{\frac{s+r}{t+r}} .
$$

Proposition 3.2. Let $B, C$ and $D$ be (not necessarily non-negative) selfadjoint operators. Then for non-negative real numbers $r, s, t$ such that $s \leq t$ and $(r, t) \neq$ $(0,0)$,

$$
\lambda B+\mu C \leq D \Rightarrow\left\{e^{\frac{r}{2} D}\left(e^{t B} ! e^{t C}\right) e^{\frac{r}{2} D}\right\}^{\frac{s+r}{t+r}} \leq e^{\frac{r}{2} D}\left(e^{s B} ! e^{s C}\right) e^{\frac{r}{2} D} .
$$

Proof. For sufficiently large $n,(1+B / n),(1+C / n)$ and $(1+D / n)$ are all nonnegative. Since $\lambda(1+B / n)+\mu(1+C / n) \leq(1+D / n)$, taking notice that

$$
(1+B / n)^{n t} !(1+C / n)^{n t} \rightarrow e^{t B} ! e^{t C} \quad \text { as } \quad n \rightarrow \infty,
$$

we can derive the desired inequality from Proposition 2.5.

By putting $s=0$ in the above remark, we get

$$
A \leq B \Rightarrow e^{r A} \leq\left(e^{\frac{r}{2} A} e^{t B} e^{\frac{r}{2} A}\right)^{\frac{r}{t+r}} .
$$

Similarly, by putting $B=C$ and $s=0$ in Proposition 3.2 we get

$$
C \leq D \Rightarrow\left(e^{\frac{r}{2} D} e^{t C} e^{\frac{r}{2} D}\right)^{\frac{r}{t+r}} \leq e^{r D} .
$$

These are known inequalities (cf. [3], 9]). Combining them, we see:

For $r, t \geq 0$ with $(r, t) \neq(0,0)$,

$$
A \leq B \leq C \Rightarrow\left(e^{\frac{r}{2} B} e^{t A} e^{\frac{r}{2} B}\right)^{\frac{r}{t+r}} \leq e^{r B} \leq\left(e^{\frac{r}{2} B} e^{t C} e^{\frac{r}{2} B}\right)^{\frac{r}{t+r}} .
$$




\section{REFERENCES}

1. W. N. Anderson, G. E. Trapp, Shorted operators II, SIAM J. Appl. Math. 28(1975), 60-71.

2. R. Bhatia, Matrix Analysis, Springer, 1996. MR 50:9417

3. M. Fujii, T. Furuta, E. Kamei, Furuta's inequality and its application to Ando's theorem, Linear Alg. Appl. 179(1993), 161-169. MR 93j:47026

4. T. Furuta, $A \geq B \geq 0$ assures $\left(B^{r} A^{p} B^{r}\right)^{1 / q}$ for $r \geq 0, p \geq 0, q \geq 1$ with $(1+2 r) q \geq p+2 r$, Proc. Amer. Math. Soc. 101(1987), 85-88. MR 89b:47028

5. T. Furuta, Two operator functions with monotone property, Proc. Amer. Math. Soc. 111(1991), 511-516. MR 91f:47023

6. F. Hansen, G. K. Pedersen, Jensen's Inequality for Operators and Löwner's Theorem, Math. Ann. 258(1982), 229-241. MR 83g:47020

7. F. Kubo, T. Ando, Means of positive linear operators, Math. Ann. 246(1980), 205-224. MR 84d:47028

8. K. Tanahashi, Best possibility of Furuta inequality, Proc. Amer. Math. Soc. 124(1996), 141146. MR 96d:47025

9. M. Uchiyama, Some exponential operator inequalities, Math. Inequal. Appl. 2(1999), 469-471. MR 2000c: 47036

10. M. Uchiyama, Strong monotonicity of operator functions, Integr. Equ. Oper. Theory 37(1) (2000), 95-105. CMP 2000:14

Department of Mathematics, Fukuoka University of Education, Munakata, Fukuoka, 811-4192, JAPAN

E-mail address: uchiyama@fukuoka-edu.ac.jp 